\title{
Spatial structure of plasma flow associated turbulence in the Earth's plasma sheet
}

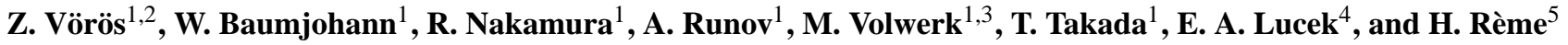 \\ ${ }^{1}$ Space Research Institute, Austrian Academy of Sciences, Graz, Austria \\ ${ }^{2}$ Institute of Atmospheric Physics, Prague, Czech Republic \\ ${ }^{3}$ Max-Plank-Institut für extraterrestrische Physik, Garching, Germany \\ ${ }^{4}$ Imperial College, London, UK \\ ${ }^{5}$ CESR/CNSR, Toulouse, France
}

Received: 17 October 2006 - Revised: 15 January 2007 - Accepted: 15 January 2007 - Published: 1 February 2007

\begin{abstract}
Using Cluster multipoint magnetic and plasma measurements we analyze the spatial structure of plasma flow-associated turbulence on 26 September 2005. The fortunate relative configuration of the spacecraft and the plasma flow allowed for the first time to compare the scale evolution of statistical moments both at the boundary and in the central part of the flow at the same time. The simultaneous increase of skewness and kurtosis at the boundary of the plasma flow over the time scale of seconds provides evidence for the existence of nonlocal coupling in flow-associated turbulence in the Earth's plasma sheet.
\end{abstract}

Keywords. Magnetospheric physics (Magnetotail; Plasma sheet) - Space plasma physics (Turbulence)

\section{Introduction}

The average properties of magnetic field and plasma within the quiet plasma sheet depend on the radial distance from the Earth, on the position relative to the neutral sheet, and also vary toward the dawn/dusk flanks (Baumjohann, 1993; Tsyganenko and Mukai, 2003). This indicates that large-scale gradients of certain quantities exist persistently along and across the Earth's magnetotail.

The whole plasma sheet undergoes significant changes during active times, especially during substorm intervals (Baumjohann, 1993). The average plasma parameters and the amplitudes of both magnetic field and velocity fluctuations vary with the level of geomagnetic activity. Previous studies indicate that the amplitude of fluctuations does not depend on the down-tail distance, nor on the distance from the neutral sheet (Neagu et al., 2002). However, neither the amplitude nor the spectral characteristics can provide

Correspondence to: Z. Vörös

(zoltan.voeroes@oeaw.ac.at) a full statistical description of fluctuations in case of turbulence. Turbulent motions are common in the plasma sheet (Borovsky and Funsten, 2003). For example, bursty bulk flow associated magnetic fluctuations exhibit multi-scale features and non-Gaussian statistics typical for turbulent flows (Vörös et al., 2003, 2004a). The frequent occurrence of fluctuations or turbulence in the plasma sheet substantiates a statistical approach including the estimation of experimentally accessible statistical moments.

In this paper higher order statistical properties of flowassociated magnetic fluctuations will be investigated. First, we introduce the basic concepts of gradient-related nonuniversal scalings in turbulence. Then we demonstrate that large-scale gradients, which are inevitably present near plasma flow boundaries, have a clear impact on the smallscale statistics in turbulence. It is known that boundaries affect higher-order statistical moments estimated from wavelet coefficients of magnetic data in the Earth's plasma sheet (Vörös et al., 2004b). However, the experimental signatures of scalar/vector gradients associated anisotropy, intermittency or direct cross-scale coupling in the Earth's plasma sheet were not studied. Here, using standard procedures (Press et al., 1992), we will directly estimate the third (skewness, $S$ ) and the fourth (kurtosis, $K$ ) statistical moments from magnetic measurements.

\section{Anisotropy and intermittency in turbulence}

Recent experimental results supported by numerical simulations indicate that small-scale anisotropy and intermittency in hydrodynamic turbulence can appear simultaneously as a consequence of specific large-scale characteristics of a flow. In particular, large-scale passive scalar gradients (e.g. temperature gradients) imposed on a turbulent velocity field induce anisotropic and intermittent fluctuations of the small-scale scalar fields (Warhaft, 2002). Accordingly, the

Published by Copernicus GmbH on behalf of the European Geosciences Union. 


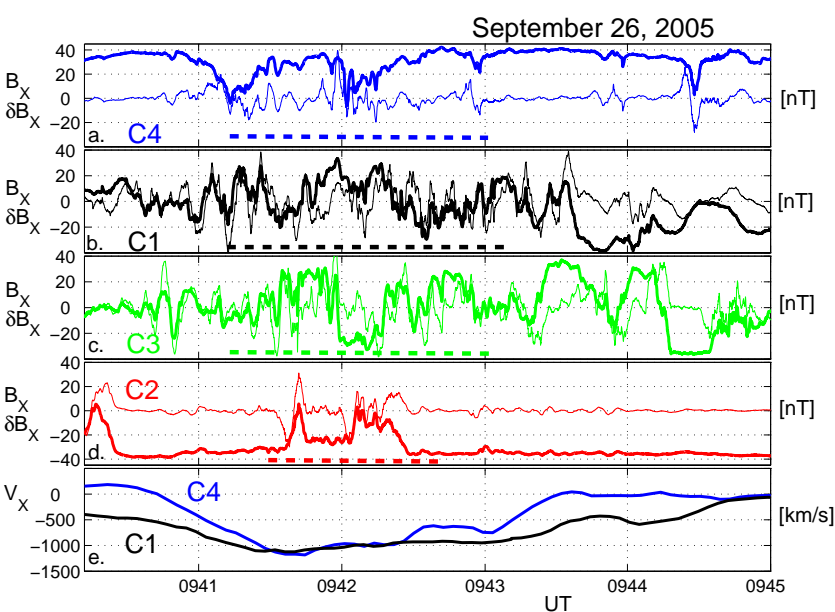

Fig. 1. Cluster magnetic and bulk velocity data. (a-d) $B_{X}$ components (thick lines) and fluctuations (thin lines) from all four spacecraft; (e) $V_{X}$ components of the bulk velocity from $\mathrm{C} 1$ and $\mathrm{C} 4$; the thick horizontal dashed lines in $(\mathrm{a}-\mathrm{d})$ show intervals of statistical moment computation.

probability density function (PDF) of the anisotropic smallscale scalar field (measured along the gradient) is skewed with asymmetric tails $(|S| \neq 0)$. At the same time, the PDF's are getting more and more peaked and long-tailed towards smaller and smaller spatial scales, a clear manifestation of intermittency (increasing $K$ ).

Large-scale gradients of the velocity (e.g. due to velocity shear) would also induce small-scale anisotropies. This case is less studied, but might be present in many hydrodynamic flow systems with velocity gradients (Warhaft, 2002).

Numerical studies show that small-scale skewed PDFs associated with large-scale scalar gradients are the manifestation of direct cross-scale coupling between well separated scales in hydrodynamic turbulence (Brethouwer et al., 2003). Another expected signature of direct cross-scale coupling is the simultaneous increase of small-scale skewness and kurtosis. In fact, statistics of a passive scalar dispersion in turbulent shear flows obey (Chatwin and Robinson, 1997)

$K=A S^{2}+B$

which is valid also, e.g. in convective and shear-dominated turbulent boundary layers in hydrodynamics (Alberghi et al., 2002). The parameters $A$ and $B$ can vary with time and position.

Nonlocal coupling in turbulence is a relatively new concept, which differs from the "standard" Kolmogorov picture, where the energy introduced at the system scale cascades towards the small scales through sequential steps - due to nonlinear, but local interactions in Fourier space. According to this picture any information about the large-scale features of a flow is lost when the cascade develops within the so-called inertial range of scales. The conceptual difference between local and nonlocal interactions entails different manners of energy redistribution in turbulence.

Magnetohydrodynamic (MHD) turbulence, however, differs in many aspects from the hydrodynamic case (Bruno and Carbone, 2005). Yet, Bershadskii and Sreenivasan (2004) have shown that in MHD flows the magnitude of the magnetic fluctuations resembles dynamical properties of passive scalars in hydrodynamics. Furthermore, Vörös et al. (2006) have shown that large-scale boundaries and/or scalar gradient fields imposed on solar wind turbulence can lead to a simultaneous increase of small-scale skewness and kurtosis, satisfying Eq. (1).

\section{Boundary turbulence on 26 September 2005}

On 26 September 2005, between 09:40 and 09:44 UT, a rather rare case occurred, when the Cluster spacecraft were merged into a tailward plasma flow, observing magnetic fluctuations not merely in the central part of the flow, but also close to the northern and southern boundaries of the flow at the same time. To illustrate the physics we describe this event in more detail first.

Figures $1 \mathrm{a}-\mathrm{d}$ show the $B_{X}$ components (thick lines) of magnetic field, available at $22 \mathrm{~Hz}$ resolution from the Flux Gate Magnetometer experiment (Balogh et al., 2001). The thin lines correspond to the fluctuations of the magnetic field, obtained through differentiation

$\delta\left(B_{X}(t, \tau)\right)=B_{X}(t+\tau)-B_{X}(t)$

where $\tau$ is the time scale of fluctuations. Supposing that the spatial fluctuations pass over the spacecraft faster than they typically fluctuate in time, the time scale $\tau$ always corresponds to a spatial scale (see further discussion in Vörös et al. (2004b)). In Fig. $1 \tau=4 \mathrm{~s}$ is used.

The subsequent panels in Figs. 1a-d show magnetic fluctuations predominantly in the Northern Hemisphere $(\mathrm{C} 4$, Fig. 1a, $B_{X}<0$ only for very short periods), in both Northern and Southern Hemispheres (C1 and C3, Figs. 1b, c), and mainly in the Southern Hemisphere (C2, Fig. 1d, $B_{X}>0$ again only for short periods). In Fig. 1e, the available 1-spin (4s) averaged velocity data from the Cluster Ion Spectrometry experiment (CIS; Réme et al., 2001) are shown from C1 (Hot Ion Analyzer) and C4 (Composition Distribution Function).

We will use the magnetic and plasma data as well as the known positions of the spacecraft (Figs. 2a-c) to figure out the approximate spatial structure of the plasma flow. The available velocity data (Fig. 1e) show that $\mathrm{C} 1$ is merged into the tailward plasma flow for a longer time than $\mathrm{C} 4$. $\mathrm{C} 1$ is almost $4 \mathrm{~min}$ in a tailward flow with $\left|V_{X}\right| \geq 500 \mathrm{~km} / \mathrm{s}$, while C4 observed such tailward flow only for $\sim 2 \mathrm{~min}$. C4 is situated Earthward from C1 (Figs. 2a, b), therefore the tailward flow should reach $\mathrm{C} 4$ earlier than $\mathrm{C} 1$. It does not happen, however, simply because before and around 09:41 UT, C4 is in 
the lobe (20nT $\leq B_{X} \leq 40 \mathrm{nT}$, Fig. 1a), while $\mathrm{C} 1$ is within the plasma sheet ( $B_{X}$ fluctuates around $0 \mathrm{nT}$, Fig. 1b). C4 enters to the plasma flow at about 09:41 UT, when $B_{X}$ starts to decrease and leaves the flow after 09:43 UT when $B_{X}$ reaches $40 \mathrm{nT}$ again. Between $\sim 09: 41$ and $\sim 09: 43$ UT, magnetic field fluctuations occur over multiple time scales from a fraction of a second up to minutes. It has already been shown that multi-scale magnetic field fluctuations always appear when bursty perpendicular plasma flows interact with the magnetic field (Vörös et al., 2004b). Since C4 enters to the flow from the northern lobe and moves back to the lobe after $\sim 2$ min, we may assume that $\mathrm{C} 4$ is situated close to the northern boundary of the plasma flow. Similarly, C2 enters to the flow from the southern lobe and appears to be close to the southern boundary of the same flow (Fig. 1d) for a relatively short time of $\sim 1 \mathrm{~min}$. Statistical analysis shows that the average width of flow channels in the north-south direction is about $10000 \mathrm{~km}$ (Nakamura et al., 2004). The distance between $\mathrm{C} 2$ and $\mathrm{C} 4$ in vertical (GSM Z) direction is only $\sim 3700 \mathrm{~km}$, however, it is easy to notice that the character of the magnetic fluctuations at $\mathrm{C} 2$ and $\mathrm{C} 4$ is similar and the flow is therefore more localized than the average width obtained from statistics. The distance between $\mathrm{C} 3, \mathrm{C} 4$ and $\mathrm{C} 1, \mathrm{C} 2$ pairs in GSM $\mathrm{X}$ direction is less than $10000 \mathrm{~km}$, which means that the tailward flow having $V_{X} \sim 1000 \mathrm{~km} / \mathrm{s}$ (C1, C4 data) travels this distance within $\sim 10 \mathrm{~s}$, while the flow itself lasts at least $2 \mathrm{~min}$. Therefore, the Cluster spacecraft are merged into the same flow. Large deviations from the mean field tend to be oriented towards the neutral sheet, and the PDFs estimated from $B_{X}$ magnetic component are skewed (not shown). Contrarily, the $C 1$ and $C 3$ spacecraft are situated roughly at the same GSM Z position (Fig. 2b), and the magnetic fluctuations in both cases are more symmetric around $B_{X}=0 \mathrm{nT}$. Both the spacecraft positions and the similarities of magnetic fluctuations at $\mathrm{C} 1$ and $\mathrm{C} 3$ as well as the available velocity data from $\mathrm{C} 1$ seem to suggest that $\mathrm{C} 1$ and C3 are close to the central part of the plasma flow. The schematic view of upper and lower boundaries of the plasma flow in GSM Y-Z plane are shown in Fig. 2c. On the basis of the relative position of spacecraft and plasma flow (Fig. 2c), we expect, that the spacecraft pairs $\mathrm{C} 2, \mathrm{C} 4$ and $\mathrm{C} 1, \mathrm{C} 3$, are situated in regions with different large-scale gradients. Near the northern/southern boundaries of the flow $(\mathrm{C} 2, \mathrm{C} 4)$ the physical parameters like density, velocity, etc. are changing rapidly back to the undisturbed or lobe level, while closer to the central part of the flow $(\mathrm{C} 1, \mathrm{C} 3)$, the gradients are expected to be smaller. Actually, we do not know how deeply C2 (or C4) get into the flow. On the other hand, optimized data intervals can be found over which the $S$ and $K$ estimates are stationary, indicating that the spacecraft are within statistically homogeneous regions. In order to check the stationarity of data intervals, $S$ and $K$ were estimated within equal number of overlapping subintervals. Then the corresponding mean values and standard deviations were computed at each scale $\tau$. Figure 3 shows the dependence of $S$ (black line) and
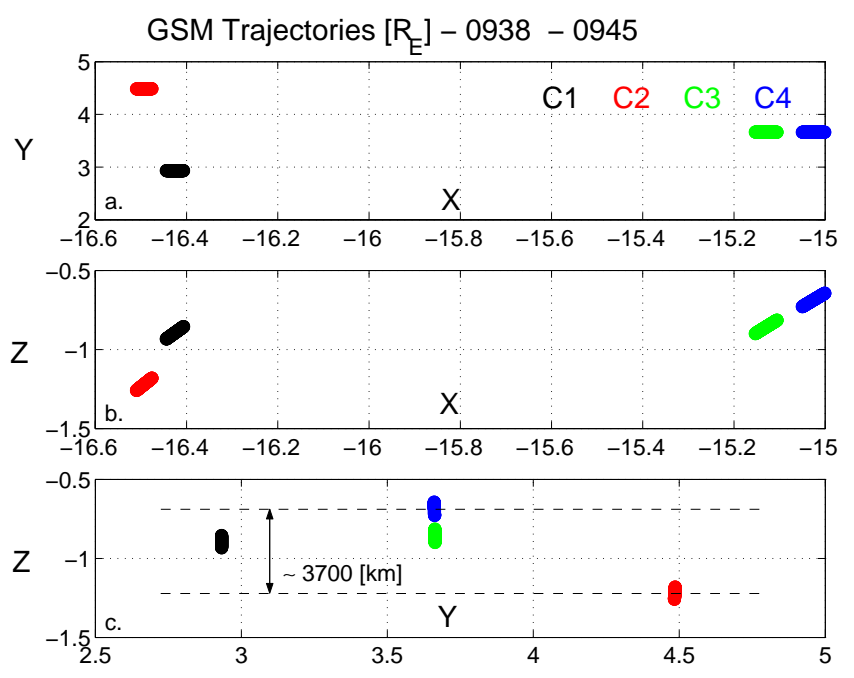

Fig. 2. Cluster spacecraft positions in GSM X-Y, X-Z and Y-Z planes. The dashed horizontal lines in (c) correspond to the approximate boundaries of the plasma flow in the Y-Z plane.

$K$ (red line) on the time scale $\tau$, estimated from the magnitude of the magnetic field. The error bars correspond to the standard deviation obtained for data intervals indicated by thick dashed horizontal lines in Figs. 1a-d. The optimized data intervals were found by trial and error. Any shifting or enlarging of the depicted intervals would increase the standard deviations over the monitored scales. Wavelet spectra exhibit multi-scale fluctuations over the intervals with the smallest standard deviation, too (not shown). The comparison of $S$ and $K$ in Figs. 3a, d (C4, C2) and 3b, c (C1, C3) shows clear differences, depending on the spacecraft position relative to the plasma flow. $\mathrm{C} 4$ and $\mathrm{C} 2$ at the flow boundary observe larger values of $S$ and $K$ above the scale of $\sim 1$ s than $\mathrm{C} 1$ and $\mathrm{C} 3$.

The scatter plot between $S$ and $K$, estimated over $2 \mathrm{~s} \leq \tau \leq 6 \mathrm{~s}$ in Fig. 4, shows interdependence of moments at the flow boundary ( $\mathrm{C} 2$ and $\mathrm{C} 4$, red points) and fluctuations around $S=K \sim 0$, within the flow (C1 and $\mathrm{C} 3$, black points). The blue curve derived using a least-squares fit corresponds to the Eq. (1) with $A=1.67 \pm 0.05$ (95\% confidence bounds) and $B=0$. In other words, we find a remarkable similarity with hydrodynamic turbulence having large-scale gradients associated with quadratic dependence of $K$ on $S$, which suggests that these signatures of nonlocal coupling occur near the boundaries of plasma flow.

\section{Conclusions}

Cluster magnetic field, velocity, and position data were used to determine the approximate spatial location of the plasma flow on 26 September 2005 relative to the Cluster spacecraft. Optimized data intervals for statistical moment estimations 


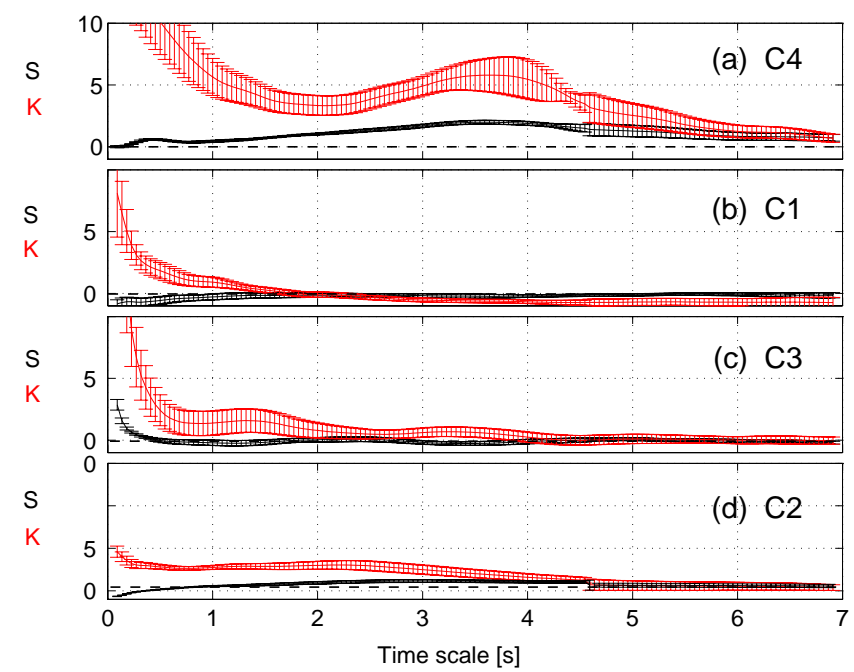

Fig. 3. Time-scale evolution of the mean values of skewness ( $S$ - black line) and kurtosis ( $K$ - red line) from all four spacecraft; the error bars correspond to standard deviations; $S$ and $K$ are estimated over the intervals indicated by thick horizontal dashed lines in Figs. 1a-d.

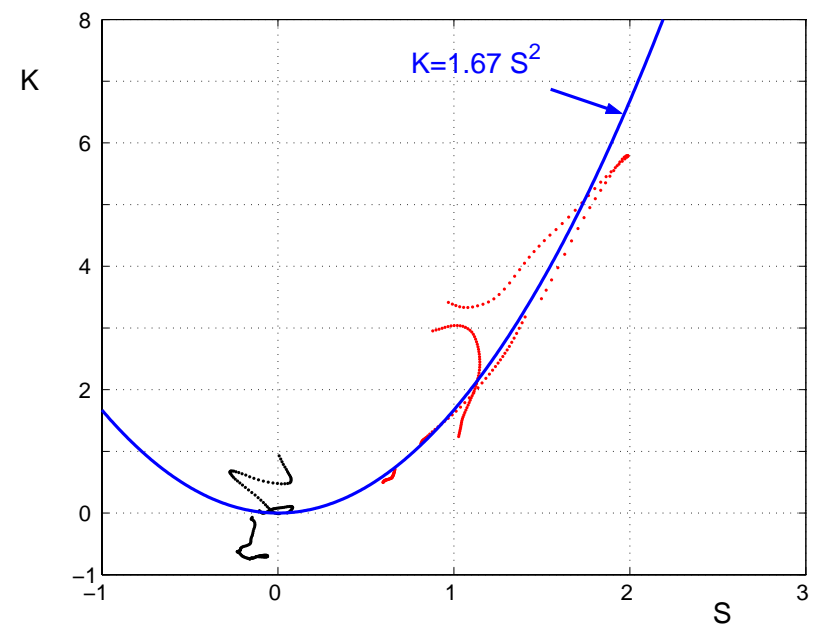

Fig. 4. Mean values of skewness versus kurtosis from $\mathrm{C} 1, \mathrm{C} 3$ (black points) and $\mathrm{C} 2, \mathrm{C} 4$ spacecraft (red points) over the time scales from 2 to $6 \mathrm{~s}$. The blue line represents the quadratic relationship of Eq. (1) with $A=1.67$ and $B=0$.

were found through trial and error. Over the time scale of seconds and at the plasma flow boundary, we found increased values of $S$ and $K$, estimated from the magnitude of magnetic fluctuations. The quadratic relationship between $S$ and $K$ resembles passive scalar statistics in turbulent shear flows or boundary layers. On this basis we can speculate that turbulence at the edges of rapid flows redistributes energy through nonlocal coupling mechanisms between wellseparated scales.
Acknowledgements. The work by Z. Vörös was partially supported by the Czech Science Foundation under project B300420509. The work by M. Volwerk was financially supported by the German Bundesministerium für Bildung und Forschung and the Zentrum für Luft- und Raumfahrt under contract 50 OC 0104.

Topical Editor I. A. Daglis thanks H. Funsten for his help in evaluating this paper.

\section{References}

Alberghi, S., Maurizi, A., and Tampieri, F.: Relationship between the vertical velocity skewness and kurtosis observed during sea breeze convection, J. Appl. Meteorol., 41, 885-889, 2002.

Balogh, A., Carr, C. M., Acuña, M. H., et al.: The Cluster magnetic field investigation: overview of in-flight performance and initial results, Ann. Geophys., 19, 1207-1217, 2001, http://www.ann-geophys.net/19/1207/2001/.

Baumjohann, W.: The near-Earth plasma sheet: an AMPTE/IRM perspective, Space Sci. Rev., 64, 141-163, 1993.

Bershadskii, A. and Sreenivasan, K. R.: Intermittency and the passive nature of the magnitude of the magnetic field, Phys. Rev. Lett., 93, 064501, doi:10.1103/PhysRevLett.93.064501, 2004.

Borovsky, J. E. and Funsten, H. O.: MHD turbulence in the Earth's plasma sheet: Dynamics, dissipation and driving, J. Geophys. Res., 108, 1284, doi:1.1029/2002JA009625, 2003.

Brethouwer, G., Hunt, J. C. R., and Nieuwstadt, F. T. M.: Microstructure and Lagrangian statistics of the scalar field with a mean gradient in isotropic turbulence, J. Fluid. Mech., 474, 193-225, 2003.

Bruno, R. and Carbone, V.: The solar wind as a turbulence laboratory, Living Rev. Solar Phys., 2, 1-186, 2005.

Chatwin, P. C. and Robinson, C.: The moments of the pdf of concentration for gas clouds in the presence of fences, Nuovo Cimento C, 20, 361- 383, 1997.

Nakamura, R., Baumjohann, W., Mouikis, C., et al.: Spatial scale of high-speed flows in the plasma sheet observed by Cluster, Geophys. Res. Lett., 31, L09804, doi:10.1029/2004GL019558, 2004.

Neagu, E., Borovsky, J. E., Thomsen, M. F., et al.: Statistical survey of magnetic field and ion velocity fluctuations in the near-Earth plasma sheet: Active Magnetospheric Particle Trace Explorers/Ion Release Module (AMPTE/IRM) measurements, J. Geophys. Res., 107(A7), 1098, doi:10.1029/2001JA000318, 2002.

Press, W. H., Teukolsky, S. A., Vetterling, W. T., and Flannery, B. P.: Numerical recipes in C, Cambridge University Press, 1992.

Réme, H., Aoustin, C., Bosqued, J. M., et al.: First multispacecraft ion measurements in and near the Earths magnetosphere with the identical Cluster ion spectrometry (CIS) experiment, Ann. Geophys., 19, 1303-1354, 2001, http://www.ann-geophys.net/19/1303/2001/.

Tsyganenko, N. A. and Mukai, T.: Tail plasma sheet models derived from Geotail particle data, J. Geophys. Res., 108(A3), 1136, doi:10.1029/2002JA009707, 2003.

Vörös, Z., Baumjohann, W., Nakamura, R., et al.: Multi-scale magnetic field intermittence in the plasma sheet, Ann. Geophys., 21, 1955-1964, 2003, http://www.ann-geophys.net/21/1955/2003/.

Vörös, Z., Baumjohann, W., Nakamura, R., et al.: Magnetic turbulence in the plasma sheet, J. Geophys. Res., 109, A11215, doi:10.1029/2004JA010404, 2004a. 
Vörös, Z., Baumjohann, W., Nakamura, R., et al.: Wavelet analysis of magnetic turbulence in the Earth's plasma sheet, Phys. Plasmas, 11, 1333-1338, doi:10.1063/1.1667499, 2004b.

Vörös, Z., Leubner, M. P., and Baumjohann, W.: Cross-scale coupling-induced intermittency near interplanetary shocks, J. Geophys. Res., 111, A02102, doi:10.1029/2005JA011479, 2006.
Warhaft, Z.: Turbulence in nature and in the laboratory, Proc. Natl. Acad. Sci. U. S. A., 99, 2481-2486, 2002. 